\title{
A set of stage-specific gene transcripts identified in EK stage $X$ and HH stage 3 chick embryos
}

\author{
Bo Ram Lee ${ }^{1}$, Heebal Kim¹, Tae Sub Park¹,2, Sunjin Moon¹, Seoae Cho3, \\ Taesung Park ${ }^{3}$, Jeong Mook Lim¹ and Jae Yong Han*1
}

\begin{abstract}
Address: ${ }^{1}$ Department of Food and Animal Biotechnology, Seoul National University, Seoul 151-921, Korea, ${ }^{2}$ Avicore Biotechnology Institute Inc., Hanlim Human Tower \#707, Gyeonggi-Do 435-050, Korea and ${ }^{3}$ Department of Statistics, Seoul National University, Seoul 151-747, Korea

Email: Bo Ram Lee - mir88@snu.ac.kr; Heebal Kim - heebal@snu.ac.kr; Tae Sub Park - taesubpark@empal.com;

Sunjin Moon - msj1547@snu.ac.kr; Seoae Cho - sacho71@snu.ac.kr; Taesung Park - tspark@stats.snu.ac.kr; Jeong Mook Lim - limjm@snu.ac.kr; Jae Yong Han* - Jaehan@snu.ac.kr

* Corresponding author
\end{abstract}

Published: I June 2007

BMC Developmental Biology 2007, 7:60

doi: $10.1|86 / 147|-213 X-7-60$
Received: 15 August 2006

Accepted: I June 2007

This article is available from: http://www.biomedcentral.com/l47I-2/3X/7/60

(C) 2007 Lee et al; licensee BioMed Central Ltd.

This is an Open Access article distributed under the terms of the Creative Commons Attribution License (http://creativecommons.org/licenses/by/2.0), which permits unrestricted use, distribution, and reproduction in any medium, provided the original work is properly cited.

\begin{abstract}
Background: The embryonic developmental process in avian species is quite different from that in mammals. The first cleavage begins $4 \mathrm{~h}$ after fertilization, but the first differentiation does not occur until laying of the egg (Eyal-Giladi and Kochav (EK) stage X). After 12 to $13 \mathrm{~h}$ of incubation (Hamburger and Hamilton $(\mathrm{HH})$ stage 3 ), the three germ layers form and germ cell segregation in the early chick embryo are completed. Thus, to identify genes associated with early embryonic development, we compared transcript expression patterns between undifferentiated (stage $\mathrm{X}$ ) and differentiated (HH stage 3) embryos.

Results: Microarray analysis primarily showed 40 genes indicating the significant changes in expression levels between stage $X$ and $\mathrm{HH}$ stage 3 , and $80 \%$ of the genes $(32 / 40)$ were differentially expressed with more than a twofold change. Among those, $72 \%(23 / 32)$ were relatively upregulated at stage $\mathrm{X}$ compared to $\mathrm{HH}$ stage 3 , while $28 \%(9 / 32)$ were relatively up-regulated at $\mathrm{HH}$ stage 3 compared to stage X. Verification and gene expression profiling of these GeneChip expression data were performed using quantitative RT-PCR for 32 genes at developmental four points; stage $X(0 \mathrm{~h}), \mathrm{HH}$ stage $3(\mathrm{I} 2 \mathrm{~h}), \mathrm{HH}$ stage $6(24 \mathrm{~h})$, and $\mathrm{HH}$ stage 9 (30 h). Additionally, we further analyzed four genes with less than twofold expression increase at $\mathrm{HH}$ stage 3 . As a result, we identified a set of stage-specific genes during the early chick embryo development; 21 genes were relatively up-regulated in the stage $X$ embryo and 12 genes were relatively up-regulated in the HH stage 3 embryo based on both results of microarray and quantitative RT-PCR.
\end{abstract}

Conclusion: We identified a set of genes with stage-specific expression from microarray Genechip and quantitative RT-PCR. Discovering stage-specific genes will aid in uncovering the molecular mechanisms involved the formation of the three germ layers and germ cell segregation in the early chick embryos. 


\section{Background}

In embryogenesis, a series of developmental events begins immediately after fertilization. During the early embryo development, the expression of many genes is spatiotemporally triggered or suppressed, under tight transcriptional control. However, the intricate changes in gene expression in the early embryo have yet to be investigated in detail in mammals or birds.

As avian species are oviparous, the embryo is readily accessible even at the earliest stages and can effectively be manipulated for purposes including profiling the genes expressed in embryogenesis. Recently, the chicken genome was completely sequenced [1], and furthermore, Burt et al [2] reported that the organization of the human genome is actually closer to that of the chicken than the mouse. Thus, the chicken is an interesting and relevant experimental animal model.

In avian species, the embryonic developmental process is quite different from that in mammals. The first cleavage begins $4 \mathrm{~h}$ after fertilization, as the embryos enter the magnum of the reproductive duct [3], but the first differentiation does not occur until the egg is laid. Cells in the embryo continue to proliferate until the Eyal-Giladi and Kochav stage $X$ and the laid egg consists of 40,000 to 60,000 undifferentiated embryonic cells [4]. After 12 to $13 \mathrm{~h}$ of incubation at Hamburger and Hamilton $(\mathrm{HH})$ stage 3 , the primitive streak extends to about the center of the area pellucida [5]. The groove in the primitive streak is gradually established as the cells of surrounding epiblast rapidly divide and migrate to the lower regions of the embryo, where they spread laterally across the surface of the yolk into two layers, the endoderm and mesoderm.

Together with when the three germ layers begin to form, the first appearance of germ cells is an important event during the early embryo development. Primordial germ cells, the progenitors of sperm or egg cells after sexual maturity, first appear from the epiblast in the blastoderm at stage $\mathrm{X}$ and translocate to the hypoblast of the area pellucida [6-8]. During gastrulation, they circulate through the vascular system and finally settle in the gonadal anlagen [9].

Understanding the molecular mechanisms that underlie germ cell segregation during early embryogenesis is important not only from the perspective of fundamental research in embryology but also from that of practical use of genetic methods. Additionally, discovering developmental stage-specific genes will aid in uncovering the molecular mechanism involved in the early stages of chicken embryo development.
In this study, we investigated the linkage of gene expression with morphological events, including germ cell segregation and identified gene transcripts from microarray GeneChip technology (stage X versus HH stage 3 ). We further analyzed our gene expression data using quantitative RT-PCR.

\section{Results \\ Microarray data analysis}

Microarray analyses were conducted to identify genes that were differentially expressed between Eyal-Giladi and Kochav stage $\mathrm{X}$ and Hamburger and Hamilton $(\mathrm{HH})$ stages 3 embryos. Raw expression levels were corrected and normalized using the RMA function and the affy software. A correlation matrix showed that the between-group variances were higher than the within-group variances (Figure 1). Hierarchical clustering was conducted upon all probes by using Cluster, and the clustering result was displayed using TreeView software (Figure 2).

Microarray analysis primarily showed 40 genes indicating the significant changes in expression levels between stage $\mathrm{X}$ and stage 3; 27 were up-regulated in the stage $\mathrm{X}$ embryo (Table 1) and 13 were up-regulated in the $\mathrm{HH}$ stage 3 embryo (Table 2). 80\% (32/40) of these genes were differentially expressed with more than a twofold change. Among those, $72 \%$ (23/32) were relatively up-regulated at stage X compared to HH stage 3 , while $28 \%(9 / 32)$ were relatively up-regulated at $\mathrm{HH}$ stage 3 compared to stage X. To verify and further characterize the GeneChip expression data, quantitative RT-PCR was performed.

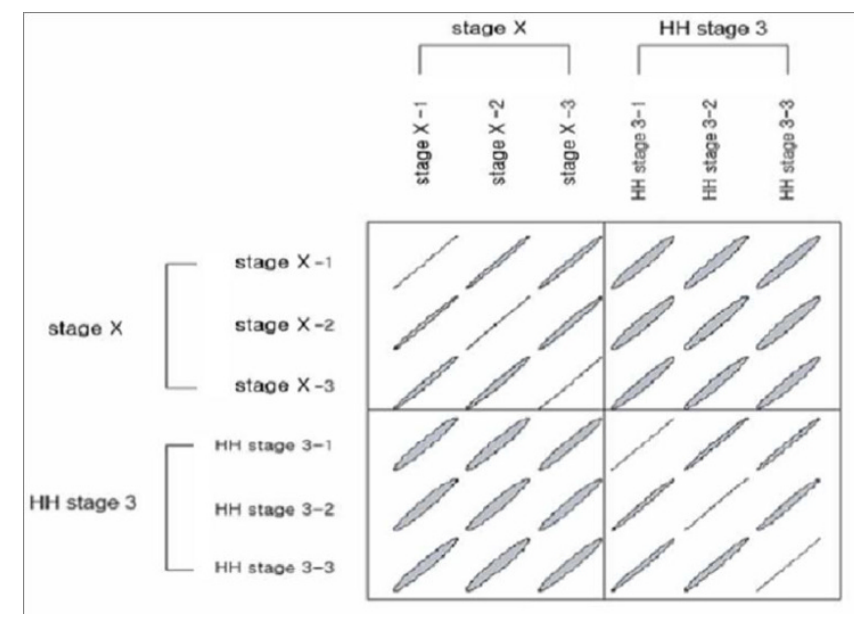

Figure I

Plot of correlation matrix between all pairs. The ellipse represents a level curve of the density of a bivariate normal with the matching correlation that shows the group variances are higher than the within variances. Each sample was performed in triplicate. 


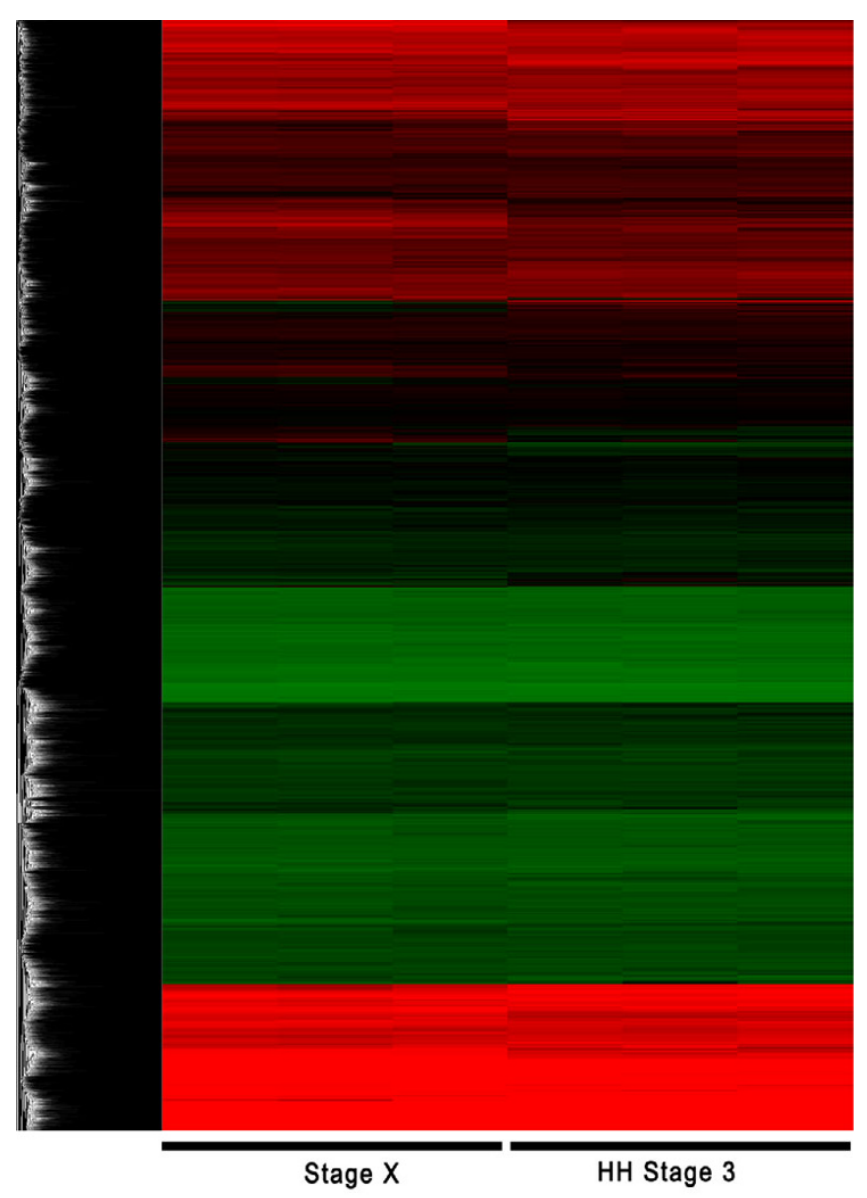

Figure 2

Clustering result of the microarray experiment. Clustering is conducted from the normalized expression data of all probes. Each row represents the relative gene expression of a single gene. Each column represents a sample. High expression relative to mean are colored red, and low expression relative to mean are colored green, and Black represents mean expression levels of the experiment. The clustering result is displayed by using TreeView software.

\section{Confirmation of differentially expressed genes via quantitative RT-PCR}

The microarray gene expression data were confirmed using quantitative RT- PCR for 32 genes showing the more than twofold changes in expression levels between stage $\mathrm{X}$ and $\mathrm{HH}$ stage 3 . Additionally, we further analyzed 4 genes with less than twofold increase in expression at $\mathrm{HH}$ stage 3 compared to stage X. Although cut-off was less than twofold, these extra genes might be important in morphological events including germ cell segregation. Thus, expression profiles of total 36 gene transcripts (23 and 13 at stage $\mathrm{X}$ and $\mathrm{HH}$ stage 3 , respectively) were quantified by RT-PCR (Table 3, 4). Furthermore, to investigate expression patterns of those transcripts during the early chick embryo development, we extended embryo development stages from stage $\mathrm{X}$ to $\mathrm{HH}$ stage 9 and prepared total mRNAs from 4 developmental points for quantitative RTPCR; stage X (0 h), HH stage 3 (12 h), HH stage 6 (24 h), and HH stage $9(30 \mathrm{~h})$. Most $(91.7 \%, 33 / 36)$ of gene transcripts were consistent with results as shown in microarray data analysis between stage $\mathrm{X}$ and $\mathrm{HH}$ stage 3. 21 genes were relatively up-regulated in the stage $\mathrm{X}$ embryo (Figure 3 ) and 12 genes were relatively up-regulated in the $\mathrm{HH}$ stage 3 embryo (Figure 4). However, 2 genes (GgaAffx.11175.1.S1_at and GgaAffx.8887.1.S1_s_at) at stage X and 1 gene (GgaAffx.11516.1.S1_s_at) at HH stage 3 showed the opposite results in quantitative PCR analysis compared to microarray data. The relative gene expression profiles of stage-specific genes were also confirmed from stage $\mathrm{X}(0 \mathrm{~h})$ to $\mathrm{HH}$ stage $9(30 \mathrm{~h})$ (Figure 3,4$)$. Based on the quantification RT-PCR results, Hierarchical clustering was performed (Figure 5).

\section{Discussion}

Recently, with chicken genome data and advances in DNA microarray technology, integration of these genomic data with the wealth of existing descriptive embryology will strengthen the position of the chicken embryo as a useful experimental model [10-13]. The chicken embryo provides an excellent model system for studying the developmental biology of vertebrates because of the accessibility of the chick embryo, allowing embryonic manipulations that are not possible in a mammalian system. But, there are few reports on microarray GeneChip technology and functional genomics for profiling gene transcripts expressed during early embryo development in avian species.

To investigate the linkage of gene expression with morphological events, including germ cell segregation, we examined differentially expressed genes between undifferentiated Eyal-Giladi and Kochav (EK) stage X and differentiated Hamburger and Hamilton ( $\mathrm{HH}$ ) stage 3 chicken embryos. Specifically, we used microarray GeneChip technology to identify a set of genes with stage-specific expression, and its results were confirmed by the relative gene expression profiling using quantitative RT-PCR. From our results based on both of microarray and quantitative RTPCR, we identified a set of stage-specific genes in the developing chick embryo: 21 genes were relatively up-regulated in stage $\mathrm{X}$ embryo and 12 genes were relatively upregulated in the $\mathrm{HH}$ stage 3 embryo.

Confirming GeneChip results with quantitative RT-PCR is important in determining whether a specific transcript is truly up- or down-regulated. Our quantitative RT-PCR results demonstrated a higher than 90\% concordance with the GeneChip data, consistent with the findings of Rajeevan et al. [14]. Their results showed a similar the 
Table I: List of the up-regulated genes in stage $\mathbf{X}$ embryo from microarray data

\begin{tabular}{|c|c|c|c|c|}
\hline$\#$ & GeneChip Probe Set ID & Gene Title & GO Molecular Function Description ${ }^{a}$ & $\log _{2} \mathrm{FCb}$ \\
\hline 1 & Gga.II040.I.SI_at & similar to hypothetical protein $\mathrm{BC} 0095 \mathrm{I} 8$ & NA & 1.208 \\
\hline 2 & Gga.I2503.2.SI_at & Nucleoredoxin & electron carrier activity & 1.874 \\
\hline 3 & Gga.I3029.I.SI_at & Finished cDNA, clone ChEST43II4 & NA & 1.561 \\
\hline 4 & Gga.I4754.I.SI_at & death associated transcription factor I & cation binding, metal ion binding & 1.514 \\
\hline 5 & Gga.I6489.I.SI_at & Finished cDNA, clone ChEST769a2I & NA & 1.111 \\
\hline 6 & Gga.I7||4.2.SI_a_at & Finished cDNA, clone ChEST485a9 & NA & 1.675 \\
\hline 7 & Gga.8833.I.SI_at & Transcribed locus & NA & 2.036 \\
\hline 8 & GgaAffx.10043.I.SI_at & $\begin{array}{l}\text { similar to Topoisomerase I binding, arginine/ } \\
\text { serine-rich }\end{array}$ & Catalytic activity & 1.498 \\
\hline 9 & GgaAffx.III75.I.SI_at & $\begin{array}{c}\text { similar to } 12 \text {-transmembrane domain co- } \\
\text { transporter Cell }\end{array}$ & Catalytic activity & 1.561 \\
\hline 10 & GgaAffx.II653.I.SI_s_at & serine/threonine kinase I7b (apoptosis-inducing) & $\begin{array}{l}\text { purine nucleotide binding, transferase activity, } \\
\text { transferring phosphorus-containing groups }\end{array}$ & 1.795 \\
\hline II & GgaAffx.21832.I.SI_s_at & fibroblast growth factor 13 & Growth factor activity & 1.822 \\
\hline 12 & GgaAffx.2367.I.SI_at & NA & NA & 1.775 \\
\hline 13 & GgaAffx.4I0I.I.SI_at & similar to transmembrane protein 20 & NA & 1.600 \\
\hline 14 & GgaAffx.4973.2.SI_s_at & similar to TNF receptor-associated factor 6 & protein binding/zinc ion binding & 2.174 \\
\hline 15 & GgaAffx.5963.I.SI_at & similar to RIKEN cDNA C030048B08 & nucleotide binding & 1.072 \\
\hline 16 & GgaAffx.8887.I.SI_s_at & similar to VANIN 3 & $\begin{array}{l}\text { hydrolase activity, acting on carbon-nitrogen (but } \\
\text { not peptide) bonds }\end{array}$ & 2.237 \\
\hline 17 & GgaAffx.9105.I.SI_at & $\begin{array}{c}\text { similar to Stromal interaction molecule } 2 \\
\text { precursor }\end{array}$ & catalytic activity/scavenger receptor activity & 1.649 \\
\hline 18 & GgaAffx.9296.3.SI_s_at & $\begin{array}{c}\text { similar to RNA polymerase II elongation factor } \\
\text { ELL2 }\end{array}$ & catalytic activity & 1.404 \\
\hline 19 & Gga.I7330.I.SI_at & splicing factor, arginine/serine-rich 15 & nucleotide binding/nucleic acid binding & 2.583 \\
\hline 20 & Gga.3I8.I.SI_at & $\begin{array}{l}\text { potassium intermediate/small conductance } \\
\text { calcium-activated channel, subfamily N, member } \\
2\end{array}$ & $\begin{array}{l}\text { alpha-type channel activity, calmodulin binding, } \\
\text { cation transporter activity, ion channel activity }\end{array}$ & 2.368 \\
\hline 21 & Gga.440I.I.SI_a_at & prostaglandin-endoperoxide synthase 2 & $\begin{array}{c}\text { cation binding, dioxygenase activity, metal ion } \\
\text { binding, oxidoreductase activity, acting on peroxide } \\
\text { as acceptor }\end{array}$ & 3.314 \\
\hline 22 & GgaAffx.20623.I.SI_s_at & LOC420789 & NA & 2.979 \\
\hline 23 & GgaAffx.7728.I.SI_at & similar to velol & NA & 2.594 \\
\hline 24 & GgaAffx.I0088.2.AI_at & similar to hypothetical protein MGC46520 & serine-type endopeptidase activity & 0.199 \\
\hline 25 & Gga.9919.I.SI_at & $\begin{array}{l}\text { similar to pre-mRNA splicing factor SF3b I55 } \\
\text { kDa subunit }\end{array}$ & Catalytic activity & 0.458 \\
\hline 26 & Gga.550.I.SI_at & $\begin{array}{c}\text { gamma-aminobutyric acid (GABA) A receptor, } \\
\text { alpha } 6\end{array}$ & $\begin{array}{c}\text { alpha-type channel activity, ion channel activity, } \\
\text { neurotransmitter receptor activity, transmembrane } \\
\text { receptor activity }\end{array}$ & 0.513 \\
\hline 27 & GgaAffx.6386.I.SI_at & similar to Ubiquitin ligase protein RNF8 & protein binding/zinc ion binding & 0.835 \\
\hline
\end{tabular}

aGene transcripts were classified using NetAffx analysis tool provided by the Affymetrix.

b $\log _{2}$ fold changes calculated from the microarray data (right column) are indicated. Log ${ }_{2} F C>I$ indicates that gene expression at stage $X$ was upregulated more than twice than that at $\mathrm{HH}$ stage 3. NA is not annotated from NetAffx analysis tool. 
Table 2: List of the up-regulated genes in $\mathrm{HH}$ stage 3 embryo from microarray data

\begin{tabular}{|c|c|c|c|c|}
\hline \# & GeneChip Probe Set ID & Gene Title & GO Molecular Function Description ${ }^{a}$ & $\log _{2} \mathrm{FC}^{\mathrm{b}}$ \\
\hline 1 & GgaAffx.2|4|3.I.SI_s_at & Finished cDNA, clone ChEST629cl3 & NA & -2.001 \\
\hline 2 & Gga.78I3.I.SI_at & Finished cDNA, clone ChESTI48dI & NA & -1.592 \\
\hline 3 & Gga.83|4.I.SI_at & Transcribed locus & NA & -1.523 \\
\hline 4 & Gga.I2I66.I.SI_at & zinc finger homeobox $\mathrm{Ib}$ & $\begin{array}{c}\text { nucleic acid binding/transcription factor activity/ } \\
\text { catalytic activity/zinc ion binding/sequence-specific } \\
\text { DNA binding }\end{array}$ & -1.474 \\
\hline 5 & Gga.|9405.I.SI_at & Finished cDNA, clone ChEST666e20 & NA & -1.318 \\
\hline 6 & GgaAffx.9643.I.SI_at & proprotein convertase PC6 & subtilase activity & -1.285 \\
\hline 7 & Gga.I6092.I.SI_at & Finished cDNA, clone ChEST357i2I & NA & -1.203 \\
\hline 8 & GgaAffx.21 I09.I.SI_s_at & Finished cDNA, clone ChESTI72i5 & guanyl-nucleotide exchange factor activity & -1.084 \\
\hline 9 & Gga.I3785.I.SI_at & heterogeneous nuclear ribonucleoprotein L-like & NA & -1.082 \\
\hline 10 & Gga.|8924.I.SI_at & Finished cDNA, clone ChEST628k5 & NA & -0.973 \\
\hline II & Gga.I5987.I.SI_at & Finished cDNA, clone ChEST48I o6 & NA & -0.662 \\
\hline 12 & GgaAffx.II5I6.I.SI_s_at & similar to septin 2 & nucleotide binding/GTP binding/ATP binding & -0.650 \\
\hline 13 & Gga.I2446.I.SI_s_at & staufen, RNA binding protein (Drosophila) & double-stranded RNA binding & -0.391 \\
\hline
\end{tabular}

aGene transcripts were classified using NetAffx analysis tool provided by the Affymetrix.

b $\log _{2}$ fold changes calculated from the microarray data (right column) are indicated. $\log _{2} \mathrm{FC}<-I$ indicates that gene expression at stage $X$ was down-regulated more than twice than that at $\mathrm{HH}$ stage 3. NA is not annotated from NetAffx analysis tool.

Table 3: List of the primer pairs for quantitative RT-PCR. Twenty-three genes were selected as being up-regulated in stage $X$ embryo from microarray data

\begin{tabular}{|c|c|c|c|c|}
\hline \# & GeneChip Probe Set ID & Gene Title & Forward & Reverse \\
\hline 1 & Gga.II040.I.SI_at & similar to hypothetical protein $\mathrm{BC} 0095 \mathrm{I} 8$ & tgctggtcaacttatgccac & gcactggaactgaaatgctg \\
\hline 2 & Gga.I2503.2.SI_at & Nucleoredoxin & gcagaacaaggtggtggg & gtggtcggaggagatgaaga \\
\hline 3 & Gga.I3029.I.SI_at & Finished cDNA, clone ChEST43II4 & tgcccttcatacctcctgac & catttattcttccaccccca \\
\hline 4 & Gga.I4754.I.SI_at & death associated transcription factor I & gacaaagcagcagagcacac & gcacccaaactggaagatg \\
\hline 5 & Gga.I6489.I.SI_at & Finished cDNA, clone ChEST769a2I & gaatcggtgtgtgacttggtt & tctccaacttccacagcaca \\
\hline 6 & Gga.I7|14.2.SI_a_at & Finished cDNA, clone ChEST485a9 & gtttcccttcccaacaggag & aaaagcccctctgattcca \\
\hline 7 & Gga.8833.I.SI_at & Transcribed locus & agggcggctttttcagatac & $\operatorname{ttgctttttgctctcctcatc}$ \\
\hline 8 & GgaAffx.I0043.I.SI_at & similar to Topoisomerase I binding, arginine/serine-rich & catcttccttcctggggact & ctgcctgtgcttcttgactg \\
\hline 9 & GgaAffx.III75.I.SI_at & similar to I2-transmembrane domain co-transporter Cell & agccttcagagagcagcaac & ttggagaacacaaagacccc \\
\hline 10 & GgaAffx.II653.I.SI_s_at & serine/threonine kinase 17b (apoptosis-inducing) & atgcttgaacagaaaacggg & ttgaacttagaaagggggca \\
\hline 11 & GgaAffx.21832.I.SI_s_at & fibroblast growth factor 13 & acagcaggcaaggataccac & aagacccaaataccaacccc \\
\hline 12 & GgaAffx.2367.I.SI_at & NA & gaacctgtaaccccgaatga & ctttccttttccgatttccc \\
\hline 13 & GgaAffx.4I0I.I.SI_at & similar to transmembrane protein 20 & tgattctcctctactatgctttcca & tcccaaacgctgtatttttctt \\
\hline 14 & GgaAffx.4973.2.sI_s_at & similar to TNF receptor-associated factor 6 & atttgagcctgccctcttct & tgtgagtgttttgcgtctcc \\
\hline 15 & GgaAffx.5963.I.SI_at & similar to RIKEN cDNA C030048B08 & tccaagaaaccagggagaag & tgactgtgattgccagaagg \\
\hline 16 & GgaAffx.8887.I.SI_s_at & similar to VANIN 3 & gaagggaaactggtggctc & cagaaagggcaagacattca \\
\hline 17 & GgaAffx.9105.I.SI_at & similar to Stromal interaction molecule 2 precursor & gggtagttatgccccgagtt & ccagtgtgcttggaggtttt \\
\hline 18 & GgaAffx.9296.3.SI_s_at & similar to RNA polymerase II elongation factor ELL2 & accaccagcaccagtcattt & gcttcttgttcatcagggga \\
\hline 19 & Gga.I7330.I.SI_at & splicing factor, arginine/serine-rich 15 & ggagccctctcaagtcactg & tgaccggagactgagctttt \\
\hline 20 & Gga.3I8.I.SI_at & $\begin{array}{l}\text { potassium intermediate/small conductance calcium-activated } \\
\text { channel, subfamily } \mathrm{N} \text {, member } 2\end{array}$ & catcttgctcggactcatca & gcgtaagaaaaggcaagtcg \\
\hline 21 & Gga.440I.I.SI_a_at & prostaglandin-endoperoxide synthase 2 & tgcaacgatatggctgagag & gggtgccagtggtacagagt \\
\hline 22 & GgaAffx.20623.I.SI_s_at & LOC420789 & tgttgaagcatacttccctt & cttgcctcttcatttgattc \\
\hline 23 & GgaAffx.7728.I.SI_at & similar to velol & accagtcccagagaacatgg & gtgattttgcccgttgactt \\
\hline
\end{tabular}

NA is not annotated from NetAffx analysis tool. 
Table 4: List of the primer pairs for quantitative RT-PCR. Thirteen genes were selected as being up-regulated in Hamburg and Hamilton (HH) stage 3 embryo from microarray data.

\begin{tabular}{|c|c|c|c|c|}
\hline$\#$ & GeneChip Probe Set ID & Gene Title & Forward & Reverse \\
\hline I & GgaAffx.2|4|3.I.SI_s_at & Finished cDNA, clone ChEST629cl3 & cccagtcccagtgtttgagt & acaagcgatgaaagaaagcc \\
\hline 2 & Gga.78I3.I.SI_at & Finished cDNA, clone ChESTI48dI & cagcacacagaaatgggaga & ctgaagaagcacacagcagg \\
\hline 3 & Gga.83|4.I.SI_at & Transcribed locus & tgtcataaacgggggaaaagtg & gctgaaagagactgtgataaaccatac \\
\hline 4 & Gga.I2166.I.SI_at & zinc finger homeobox $\mathrm{Ib}$ & aagtcgagtgcaattcgtaca & tggactaaagggggcagttca \\
\hline 5 & Gga.I9405.I.SI_at & Finished cDNA, clone ChEST666e20 & tgctctgtccttcttctgcc & ccccaaccatctctccagt \\
\hline 6 & GgaAffx.9643.I.SI_at & proprotein convertase PC6 & tcttcttcaactgcctgcct & gaacaccgtccttcgtcact \\
\hline 7 & Gga.I6092.I.SI_at & Finished cDNA, clone ChEST357i2I & ttggcaggaaggaagacaag & gcaactctacgaactggctg \\
\hline 8 & GgaAffx.21 109.I.SI_s_at & Finished cDNA, clone ChESTI72i5 & ggttggaatcttgtggcagt & gcttgttggtcggtcttctc \\
\hline 9 & Gga.|3785.I.SI_at & heterogeneous nuclear ribonucleoprotein L-like & gctttcatcaggttaatgttgc & catgctaatgcaaacggaaa \\
\hline 10 & Gga.I8924.I.SI_at & Finished cDNA, clone ChEST628k5 & accacaggcagtacggctac & ccccaaggaaaatttaaaacg \\
\hline 11 & Gga.I5987.I.SI_at & Finished cDNA, clone ChEST48I o6 & tcataccagtctcaagcaggg & tccttcattcctcactcaaaca \\
\hline 12 & GgaAffx.II5I6.I.SI_s_at & similar to septin 2 & acttccgttcagagaggctg & agcacccgtccacttagaga \\
\hline 13 & Gga.I2446.I.SI_s_at & staufen, RNA binding protein (Drosophila) & ccgcagattgattttccttg & acttgcaaggctatcgtgct \\
\hline
\end{tabular}

degree of agreement between GeneChip and QRT-PCR and that the agreement was related to hybridization intensity, with the highest level of concordance for those genes with the highest signal intensity on the GeneChip. From the quantitative RT-PCR in this study, most $(21 / 23)$ of genes up-regulated in stage $\mathrm{X}$ embryo gradually decreased according to developmental process, while almost (12/ 13) of genes up-regulated in HH stage 3 embryo were relatively higher than in stage $\mathrm{X}$ and increased according to ages of incubation time until $\mathrm{HH}$ stage $9(30 \mathrm{~h})$. However, three gene transcripts (2 at stage $\mathrm{X}$ and 1 at HH stage 3 ) showed the discrepancy between microarray data and the quantitative RT-PCR analysis. Thus, further intensive experiments such as Northern blot and in situ hybridization as well as functional study would be conducted.

The freshly laid egg, at stage $\mathrm{X}$, consists of 40,000 to 60,000 undifferentiated embryonic cells. At this stage, notably, anti-oxidation-related transcripts such as Gga.12503.2.S1_at (Nucleoredoxin) and Gga.4401.1.S1_a_at (prostaglandin-endoperoxide synthase 2) were expressed higher than at $\mathrm{HH}$ stage 3 . Oxidative stress is one of the main damage sources in living organisms, and the anti-oxidant defence mechanisms of biological systems play major roles in preventing oxidative stress and the consequent cell damage [15]. Thus, higher expression of anti-oxidation-related transcripts could be one of the critical mechanisms for survival at stage $\mathrm{X}$ embryo.

Signal transduction pathways are known to play key roles in cell fate commitments during early embryogenesis. FGF13, highly expressed in stage X embryo, would be involved in the signalling transduction during the early chick embryogenesis. FGF signaling not only mediates cell fate specification in the early embryo but also maintains an undifferentiated cell state in many cellular contexts [16]. Streit et al [17] suggested that FGF secreted from a population of organizer precursors initiates induction of neural system before the beginning of gastrulation and Sato et al [18] reported that in human embryonic stem cells, FGF2/13 expression is enriched and important to understand the molecular mechanisms underlying stemness.

At HH stage 3 (12 h of incubation), three germ layers and germline segregation with the appearance of primordial germ cells (PGCs) begin to form in chick embryogenesis. Since the number of these PGCs is rare at the early developmental stags, it would be difficult to investigate the germline-specific genes. In this study, 12 gene transcripts which were relatively up-regulated in $\mathrm{HH}$ stage 3 embryos might be important in germ cell segregation as well as morphological events. Interestingly, Gga.12446.1.S1_s_at, transcript up-regulated at HH stage 3, was gallus gallus staufen homolog to Drosophila STAU1 and gradually increased during developmental process. Staufen, a double-stranded RNA binding protein, was first identified in Drosophila as an essential factor required for germline specification and the anterior-posterior axis formation [19]. Ramasamy et al. [20] recently reported that Zebra fish Staufen 1 and Staufen 2 are required for the survival and migration of primordial germ cells.

Septin2 (GgaAffx.11516.1.S1_s_at), GTP-binding protein, is involved in cytokinesis in various species from yeast and vertebrates [21]. Expression level of Septin2 was significantly found in mouse embryonic heart in a developmentally regulated fashion and gradually down-regulated around birth [21]. In our GeneChip data, Septin2 were expressed higher at $\mathrm{HH}$ stage 3 than at stage $\mathrm{X}$ and it might be that embryonic cells actively proliferate and organogenesis begins during gastrulation. However, the results from quantitative RT-PCR showed septin2 transcript consistently expressed from stage $\mathrm{X}$ to HH stage 9. Thus, spatiotemporal regulation(s) of septin2 transcript expression remain to be further investigated. 


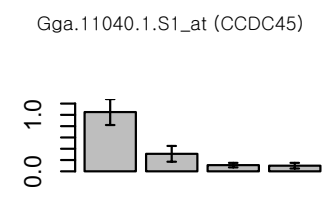

Oh $12 \mathrm{~h} \quad 24 \mathrm{~h} \quad 30 \mathrm{~h}$

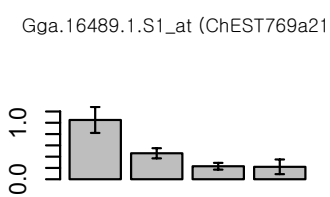

Oh $12 \mathrm{~h} \quad 24 \mathrm{~h} \quad 30 \mathrm{~h}$

GgaAffx.11175.1.S1_at (LOC431636)

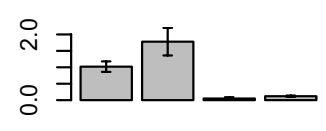

Oh $12 \mathrm{~h} \quad 24 \mathrm{~h} \quad 30 \mathrm{~h}$

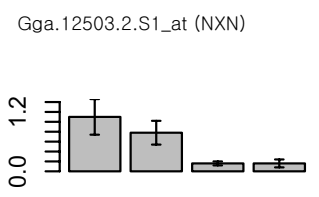

Oh $12 \mathrm{~h} \quad 24 \mathrm{~h} \quad 30 \mathrm{~h}$

Gga.17114.2.S1_a_at (ChEST485a9)

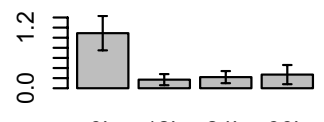

Oh $12 \mathrm{~h} \quad 24 \mathrm{~h} \quad 30 \mathrm{~h}$
Gga.13029.1.S1_at (ChEST43/14)

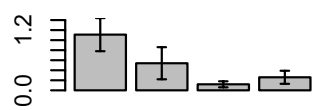

Oh $12 \mathrm{~h} \quad 24 \mathrm{~h} \quad 30 \mathrm{~h}$
Gga.14754.1.S1_at (RCJMB04_5H22)

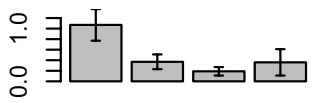

Oh $12 \mathrm{~h} \quad 24 \mathrm{~h} \quad 30 \mathrm{~h}$
Gga.8833.1.S1_at (Transcribed locus)

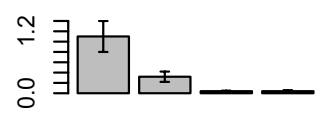

Oh $12 \mathrm{~h} \quad 24 \mathrm{~h} \quad 30 \mathrm{~h}$
GgaAffx.10043.1.S1_at (LOC427342)

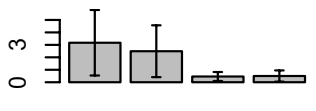

Oh $12 \mathrm{~h} \quad 24 \mathrm{~h} \quad 30 \mathrm{~h}$
GgaAffx.4101.1.S1_at (LOC423800)

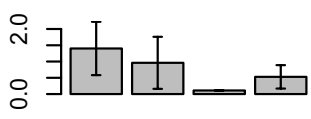

Oh $12 \mathrm{~h} \quad 24 \mathrm{~h} \quad 30 \mathrm{~h}$

GgaAffx.9105.1.S1_at (LOC422799)

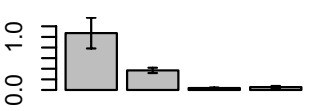

Oh $12 \mathrm{~h} \quad 24 \mathrm{~h} \quad 30 \mathrm{~h}$
GgaAffx.4973.2.S1_s_at (LOC423163)

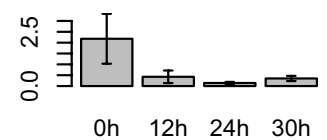

GgaAffx.9296.3.S1_s_at (LOC427119)

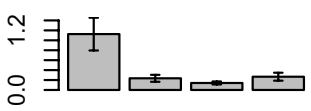

Oh $\quad 12 \mathrm{~h} \quad 24 \mathrm{~h} \quad 30 \mathrm{~h}$
GgaAffx.5963.1.S1_at (RCJMB04_7N4)

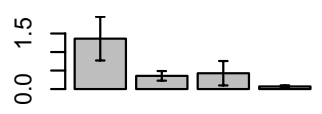

Oh $12 \mathrm{~h} \quad 24 \mathrm{~h} \quad 30 \mathrm{~h}$

Gga.17330.1.S1_at (RCJMB04_3H16)

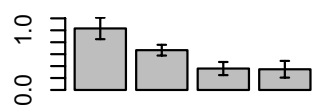

Oh $12 \mathrm{~h} \quad 24 \mathrm{~h} \quad 30 \mathrm{~h}$
GgaAffx.2367.1.S1_at (NA)

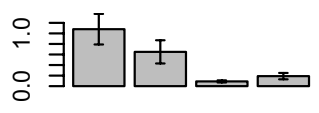

Oh $12 \mathrm{~h} \quad 24 \mathrm{~h} \quad 30 \mathrm{~h}$
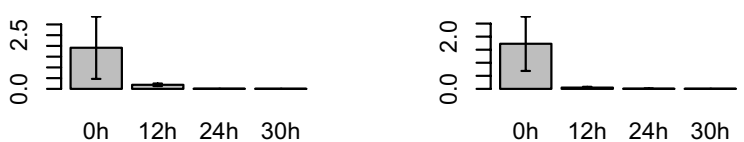

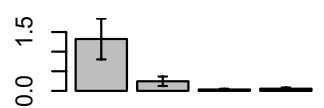

Oh $12 \mathrm{~h} \quad 24 \mathrm{~h} \quad 30 \mathrm{~h}$
GgaAffx.8887.1.S1_s_at

GgaAffx.8887.1.S1_s_at
(RCJMB04_35G11)

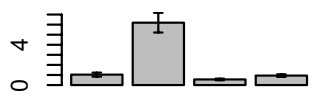

Oh $12 \mathrm{~h} \quad 24 \mathrm{~h} \quad 30 \mathrm{~h}$

Gga.318.1.S1_at (KCNN2)

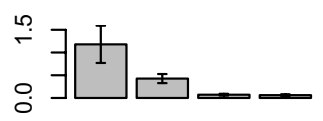

Oh $\quad 12 \mathrm{~h} \quad 24 \mathrm{~h} \quad 30 \mathrm{~h}$

\section{Figure 3}

Quantification of the relative expression of the 23 genes selected as being up-regulated in stage $X$ embryo compared to Hamburg and Hamilton (HH) stage 3. Quantitative RT-PCR was conducted at stage $\mathrm{X}(\mathrm{O}$ h), $\mathrm{HH}$ stage 3 (I $2 \mathrm{~h})$, $\mathrm{HH}$ stage $6(24 \mathrm{~h})$, and $\mathrm{HH}$ stage $9(30 \mathrm{~h})$ embryos. The relative quantification of gene expression was analyzed by the 2$\Delta \Delta \mathrm{Ct}_{\mathrm{t}}$ method. NA is not annotated from NetAffx analysis tool. 
GgaAffx 21413.1.S1_s_at

(ChEST629c13)

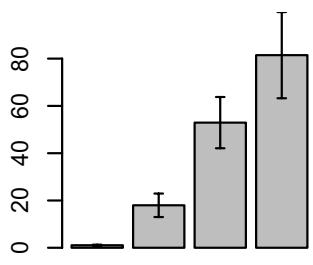

Oh 12h 24h $30 \mathrm{~h}$

Gga.19405.1.S1_at (ChEST666e20)

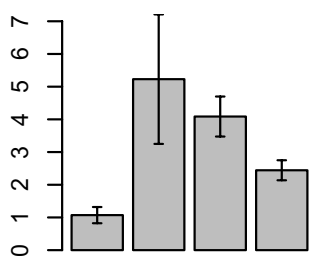

Oh $12 \mathrm{~h} \quad 24 \mathrm{~h} \quad 30 \mathrm{~h}$

Gga.13785.1.S1_at (HNRPLL)

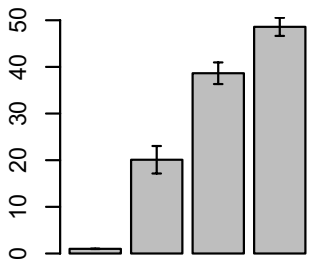

oh $12 \mathrm{~h} 24 \mathrm{~h} 30 \mathrm{~h}$

Gga.12446.1.S1_s_at (RCJMB04_32C1)

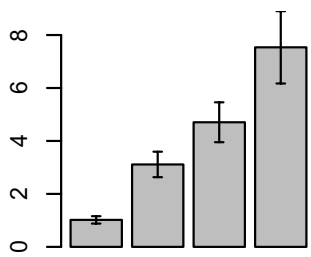

Oh 12h 24h $30 \mathrm{~h}$
Gga.7813.1.S1_at

(ChEST148d1)

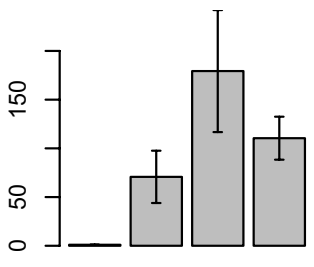

Oh $12 \mathrm{~h} \quad 24 \mathrm{~h} \quad 30 \mathrm{~h}$

GgaAffx.9643.1.S1_at (PC6)

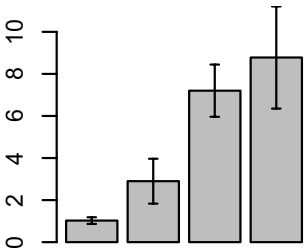

Oh $12 \mathrm{~h} \quad 24 \mathrm{~h} \quad 30 \mathrm{~h}$

Gga.18924.1.S1_at (ChEST628k5)

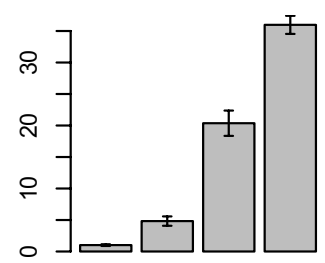

Oh $12 \mathrm{~h} \quad 24 \mathrm{~h} \quad 30 \mathrm{~h}$
Gga.8314.1.S1 at

(Transcribed locus)

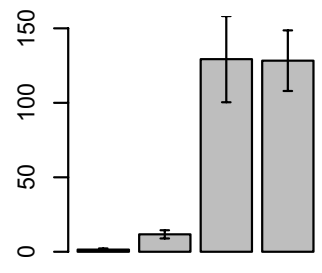

Oh $12 \mathrm{~h} \quad 24 \mathrm{~h} \quad 30 \mathrm{~h}$

Gga.16092.1.S1_at (ChEST357i21)

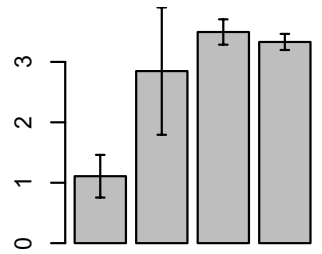

oh $12 \mathrm{~h} \quad 24 \mathrm{~h} \quad 30 \mathrm{~h}$

Gga.15987.1.S1_at

(ChEST48106)

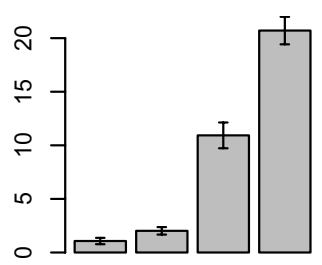

Oh $12 \mathrm{~h} \quad 24 \mathrm{~h} \quad 30 \mathrm{~h}$
Gga.12166.1.S1 at (ZFHX1B)

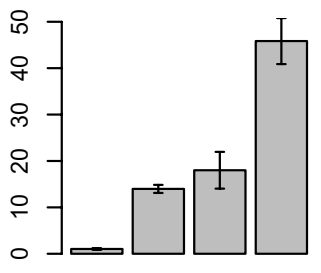

Oh $12 \mathrm{~h} \quad 24 \mathrm{~h} \quad 30 \mathrm{~h}$

GgaAffx.21109.1.S1_s_at (ChEST172i5)

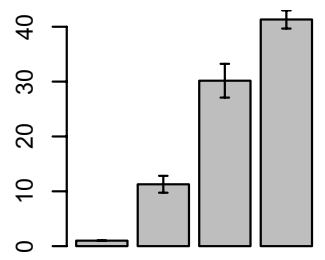

Oh $12 \mathrm{~h} \quad 24 \mathrm{~h} \quad 30 \mathrm{~h}$

GgaAffx.11516.1.S1_s_at (LOC416777)

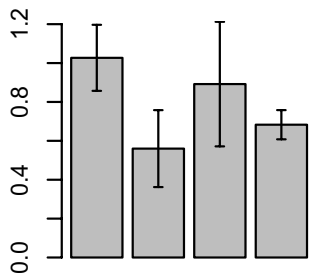

Oh $12 \mathrm{~h} \quad 24 \mathrm{~h} \quad 30 \mathrm{~h}$

Figure 4

Quantification of the relative expression of the 13 genes selected as being up-regulated in Hamburg and Hamilton (HH) stage 3 embryo compared to stage X. Quantitative RT-PCR was conducted at stage X (0 h), HH stage 3 (I 2 h), $\mathrm{HH}$ stage $6(24 \mathrm{~h}), \mathrm{HH}$ stage $9(30 \mathrm{~h})$ embryos. The relative quantification of gene expression was analyzed by the 2 - $\Delta \Delta \mathrm{Ct}$ method. 


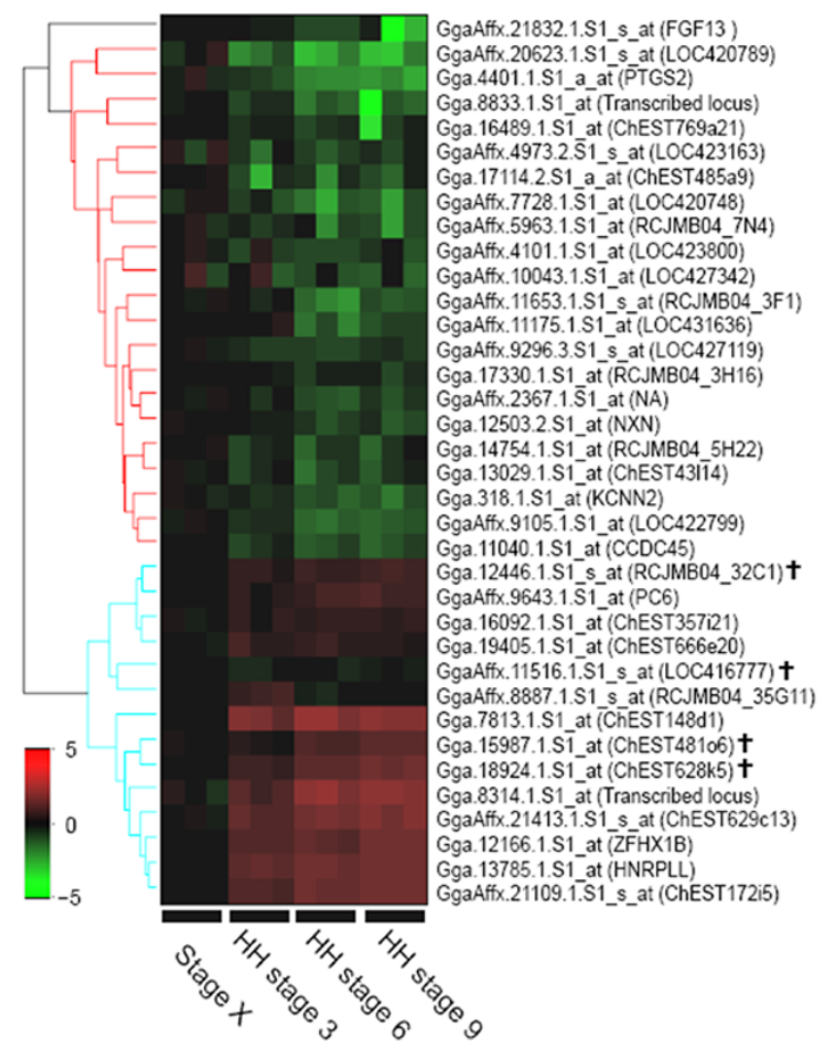

Figure 5

Hierarchical clustering of differentially expressed genes analyzed by quantitative RT-PCR. Based on the microarray analysis, the 23 genes selected as being up-regulated at stage $X$ and the 13 genes selected as being up-regulated at $\mathrm{HH}$ stage 3 were confirmed by quantitative RT-PCR at stage $X(0 \mathrm{~h}), \mathrm{HH}$ stage $3(\mathrm{I} 2 \mathrm{~h}), \mathrm{HH}$ stage $6(24 \mathrm{~h})$, and $\mathrm{HH}$ stage $9(30 \mathrm{~h})$. Most $(91.7 \%, 33 / 36)$ of genes were consistent with results as shown of microarray data analysis. Color indicates the relative expression levels of each gene, with red indicating higher expression, green indicating negative expression. Black represents a control group that is normalized for the relative quantification of gene expression. Four genes with less than twofold expression increase at $\mathrm{HH}$ stage 3 were indicated (). NA is not annotated from NetAffx analysis tool.

Although the number of genes analyzed in this study was limited, our data would be contributed to understanding how developmentally important genes are integrated with developmental processes and germline segregation during early embryogenesis in the chicken. Further experiments examining in situ cellular expression and functional properties of the identified genes would be needed to elucidate the transcriptional control mechanisms of gene expression associated with early embryogenesis in chickens.

\section{Conclusion}

In this study, we identified a set of genes with stage-specific expression using microarray Genechip and quantitative RT-PCR during the early embryo development in avian species. Discovering stage-specific genes will aid in uncovering the molecular mechanisms involved the formation of the three germ layers and germ cell segregation in the early chick embryos.

\section{Methods \\ Sample preparation and RNA extraction}

Experimental animals for this study were maintained at the University Animal Farm, Seoul National University, Korea. All experimental procedures were performed at the affiliated laboratories of the university.

Eggs were brought to the lab within 1 to $3 \mathrm{~h}$ of oviposition for stage X embryos [4]. Developing embryos under relative humidity of $60-70 \%$ at $37.8^{\circ} \mathrm{C}$ were staged according to the Hamburger and Hamilton $(\mathrm{HH})$ classification system [6]. Thus, Eyal-Giladi and Kochav (EK) stages X, Hamburg and Hamilton ( $\mathrm{HH}$ ) stage $3, \mathrm{HH}$ stage 6 , and $\mathrm{HH}$ stage 9 correspond to embryos at 0 and $12 \mathrm{~h}, 24 \mathrm{~h}$, and $30 \mathrm{~h}$ of incubation, respectively.

Total RNA from embryos at each stage was isolated using the TRIzol Reagent (Invitrogen, Carlsbad, CA), according to the manufacturer's instructions. RNA quality was checked by agarose gel electrophoresis and RNA quantity was determined by spectrophotometry at $260 \mathrm{~nm}$. RNA was then further purified using the RNeasy kit (Qiagen, Valencia, CA). The total RNA was used for gene expression analysis on an Affymetrix GeneChip Chicken Genome Array (Affymetrix, Santa Clara, CA), containing 38,535 probes.

\section{Generation of microarray data}

All experiments were performed in triplicate. The generation of GeneChip data from the embryos of stage $\mathrm{X}$ and $\mathrm{HH}$ stages 3 was performed by Seoulin Bioscience Corporation (Seoul, Korea). Specifically, total RNA (about $5 \mu \mathrm{g}$ ) from stage $\mathrm{X}$ and $\mathrm{HH}$ stages 3 embryos was used for labelling. Probe synthesis from total RNA samples, hybridization, detection, and scanning were performed according to standard protocols from Affymetrix. Briefly, cDNA was synthesized using the One-Cycle cDNA Synthesis Kit (Affymetrix). Single-stranded (ss) cDNA was synthesized using Superscript II reverse transcriptase and T7-oligo (dT) primers at $42^{\circ} \mathrm{C}$ for $1 \mathrm{~h}$. Double-stranded (ds) cDNA was obtained using DNA ligase, DNA polymerase I, and RNase $\mathrm{H}$ at $16^{\circ} \mathrm{C}$ for $2 \mathrm{~h}$, followed by T4DNA polymerase at $16^{\circ} \mathrm{C}$ for $5 \mathrm{~min}$. After cleanup using a Sample Cleanup Module (Affymetrix, Santa Clara, CA), ds cDNA was used for in vitro transcription (IVT). cDNA was transcribed using the GeneChip IVT Labeling Kit (Affymetrix) in the 
presence of biotin-labeled CTP and UTP. Then the biotinlabeled IVT-RNA was fragmented and hybridized to the chicken genome GeneChip array at $45^{\circ} \mathrm{C}$ for $16 \mathrm{~h}$, according to the manufacturer's instructions. After hybridization, the arrays were washed in a GeneChip Fluidics Station 450 with a non-stringent wash buffer at $25^{\circ} \mathrm{C}$, followed by a stringent wash buffer at $50^{\circ} \mathrm{C}$. After washing, the arrays were stained with a streptavidin-phycoerythrin complex. After staining, intensities were determined with a GeneChip scanner, controlled by GeneChip Operating Software (GCOS; Affymetrix).

\section{Microarray data analysis}

The quality of the array image was assessed as described in the GeneChip expression analysis manual (Affymetrix). All arrays were processed to determine the "robust multiarray average" (RMA; [22]) using the "affy" software package [23]. Expression values were computed in detail from raw CEL files by applying the RMA model of probe-specific correction for perfect-match probes. These corrected probe values were then subjected to quantile normalization, and a median polish was applied to compute one expression measure from all probe values. Resulting RMA expression values were $\log _{2}$-transformed. Clustering from the normalized expression data of all probes was performed and displayed using Cluster and TreeView software [24].

Individual gene expression levels from embryos at stage $\mathrm{X}$ and HH stages 3 were compared using an unpaired Student's $t$-test. The Benjamini-Hochberg correction for false discovery rate (FDR) was used for all probe-level normalized data. We selected differentially expressed genes that met the following criteria: a change in expression of at least twofold when comparing the means of the two groups and a FDR-adjusted $P$ value of less than 0.01 according to the unpaired Student's $t$-test. Gene Ontology annotation was conducted using NetAffx tool provided by the Affymetrix [25]. Further analysis for functional annotation clustering was performed using the DAVID database [26] [see Additional file 1].

\section{Quantitative RT-PCR}

To confirm the GeneChip expression data and the relative gene expression profiling, quantitative RT-PCR was performed at the developmental stages. Based on microarray data, we selected 36 genes and primers were designed using the Primer 3 software [27] on sequences from the GenBank database (Table 3, 4). For quantitative RT-PCR, we extended embryo development stages from stage $\mathrm{X}$ to stage 9 and so prepared total mRNAs from 4 developmental points; stage $\mathrm{X}(0 \mathrm{~h}), \mathrm{HH}$ stage $3(12 \mathrm{~h})$, HH stage 6 (24 $\mathrm{h})$, and HH stage $9(30 \mathrm{~h})$. sscDNA was synthesized from total RNA $(1 \mu \mathrm{g})$ using the Superscript III First-Strand Synthesis System (Invitrogen, Carlsbad, CA). The cDNA was serially diluted fivefold and was quantitatively equalized for PCR amplification. Quantitative RT-PCR was performed using the iCycler iQ real-time PCR detection system (Bio-Rad Laboratories, Hercules, CA) and SYBR Green I (Sigma, St. Louis, MO). The glyceraldehyde-3phosphate dehydrogenase (GAPDH) gene was run simultaneously as a control and used for normalization. Nontemplate control wells with no cDNA were included as negative controls. Each test sample was run in triplicate.

Following the standard curve method, the expression quantities of the examined genes were determined using the standard curves and the Ct values, and were normalized using GAPDH expression quantities. The PCR conditions were $94^{\circ} \mathrm{C}$ for $3 \mathrm{~min}$, followed by 40 cycles at $94^{\circ} \mathrm{C}$ for $30 \mathrm{sec}, 60^{\circ} \mathrm{C}$ for $30 \mathrm{sec}$, and $72^{\circ} \mathrm{C}$ for $30 \mathrm{sec}$, using a melting curve program (increasing temperature from 55 to $95^{\circ} \mathrm{C}$ with a heating rate of $0.5^{\circ} \mathrm{C}$ per $10 \mathrm{sec}$ ) and continuous fluorescence measurement. Sequence-specific products were identified by generating a melting curve. The Ct value represents the cycle number at which a fluorescent signal rises statistically above background and the relative quantification of gene expression was analyzed by the $2^{-\Delta \Delta \mathrm{Ct}}$ method [28]. Based on the quantification RTPCR results, hierarchical clustering was performed, and the relative quantification of gene expression was normalized to the Ct of stage $\mathrm{X}$ as a control group.

\section{Authors' contributions}

BRL carried out all the microarray experiment and quantitative RT-PCR, HK, SC and TP carried out the microarray data analysis, JYH, JML and HK conceived and coordinated the study, and BRL and TSP wrote the manuscript. Figures and quantitative RT-PCR results were prepared by BRL and SM. All authors read and approved the final manuscript.

\section{Additional material}

\section{Additional File 1}

Functional annotation clustering of a set of genes identified from microarray experiment. This data represent 5 term clusters generated from functional annotation clustering of total 40 genes identified from microarray results using DAVID database provided by NIAID.

Click here for file

[http://www.biomedcentral.com/content/supplementary/1471-

213X-7-60-S1.xls]

\section{Acknowledgements}

We thank Seoulin Bioscience Corporation (Seoul, Korea) for Genechip data generation. This work is supported in part by a grant from BioGreen 2I Program of the Rural Development Administration and by the Brain Korea 2I Project of the Ministry of Education. The work of Taesung Park 
was partially supported by the National Research Laboratory Program of Korea Science and Engineering Foundation.

\section{References}

I. Schmutz J, Grimwood J: Genomes: Fowl sequence. Nature 2004, 432:679-680.

2. Burt DW, Bruley C, Dunn IC, Jones CT, Ramage A, Law AS, Morrice DR, Paton IR, Smith J, Windsor D, Sazanov A, Fries R, Waddington D: The dynamics of chromosome evolution in birds and mammals. Nature 1999, 402:4II-4I3.

3. Perry MM: Nuclear events from fertilisation to the early cleavage stages in the domestic fowl (Gallus domesticus). J Anat 1987, 150:99-109.

4. Eyal-Giladi H, Kochav S: From cleavage to primitive streak formation: a complementary normal table and a new look at the first stages of the development of the chick. I. General morphology. Dev Biol 1976, 49:32I-370.

5. Wei Y, Mikawa T: Formation of the avian primitive streak from spatially restricted blastoderm: evidence for polarized cel division in the elongating streak. Development 2000, 1 27:87-96.

6. Hamburger $\mathrm{V}$, Hamilton HL: A series of normal stages in the development of the chick embryo. J Morphol I95I, 88:49-92.

7. Nieuwkoop PD, Sutasurya LA: Primordial germ cells in the Chordates. Cambridge University Press, Cambridge; 1979.

8. Ginsburg $M$, Eyal-giladi $\mathrm{H}$ : Temporal and spatial aspects of the gradual migration of primordial germ cells from the epiblast into the germinal crescent of the avian embryo. J Embryol Exp Morpho 1986, 95:53-7I.

9. Swift $\mathrm{CH}$ : Origin and early history of the primordial germ cells in the chick. Am J Anat 1914, 15:483-516.

10. Itasaki N, Bel-Vialar S, Krumlauf R: 'Shocking' developments in chick embryology: electroporation and in ovo gene expression. Nature Cell Biol 1999, I(8):E203-E207.

II. Ko MS: Embryogenomics: developmental biology meets genomics. Trends Biotechnol 200I, I9:5 I I-5 I8.

12. Pekarik V, Bourikas D, Miglino N, Joset P, Preiswerk S, Stoeckli ET: Screening for gene function in chicken embryo using RNAi and electroporation. Nature Biotechnol 2003, 2 I (I):93-96.

13. Brown WR, Hubbard SJ, Tickle C, Wilson SA: The chicken as a model for large-scale analysis of vertebrate gene function. Nature Rev 2003, 4(2):87-98.

14. Rajeevan MS, Vernon SD, Taysavang N, Unger ER: Validation of array-based gene expression profiles by real-time (kinetic) RT-PCR. J Mol Diagn 200I, 3:26-3I.

15. Han JY, Song KD, Shin JH, Han BK, Park TS, Park HJ, Kim JK, Lillehoj $\mathrm{HS}$, Lim JM, Kim H: Identification and characterization of the peroxiredoxin gene family in chickens. Poult Sci 2005, 84: |432-1438

16. Delfino-Machín M, Lunn JS, Breitkreuz DN, Akai J, Storey KG: Specification and maintenance of the spinal cord stem zone. Development 2005, I 32:4273-4283.

17. Streit A, Berliner AJ, Papanayotou C, Sirulnik A, Stern CD: Initiation of neural induction by FGF signalling before gastrulation. Nature 2000, 406:74-78.

18. Sato N, Sanjuan IM, Heke M, Uchida M, Naef F, Brivanlou AH: Molecular signature of human embryonic stem cells and its comparison with the mouse. Dev Biol 2003, 260:404-4I3.

19. St Johnston D, Beachle D, Nusslein-Volhard C: Staufen, a gene required to localize maternal RNAs in the Drosophila egg. Cell 199I, 66:5I-63.

20. Ramasamy S, Wang H, Quach HN, Sampath K: Zebrafish Staufen I and Staufen 2 are required for the survival and migration of primordial germ cells. Dev Bio 2006, 292(2):393-406.

21. Ahuja P, Perriard E, Trimble W, Perriard JC, Ehler E: Probing the role of septins in cardiomycytes. Exp Cell Res 2006 3 I2:1598-1609.

22. Irizarry RA, Bolstad BM, Collin F, Cope LM, Hobbs B, Speed TP: Summaries of Affymetrix GeneChip probe level data. Nucleic Acids Res 2003, 3 I:el 5.

23. Gautier L, Cope L, Bolstad BM, Irizarry RA: affy--analysis of Affymetrix GeneChip data at the probe-level. Bioinformatics 2004, 20:307-315.

24. Cluster Analysis Software [http://rana.lbl.gov/EisenSoft ware.htm]
25. NetAffx Analysis Center [http://www.affymetrix.com/analysis/ index.affx]

26. DAVID [http://david.abcc.ncifcrf.gov]

27. Rozen S, Skaletsky H: Pimer3 on the WWW for general users and for biologist programmers. Methods Mol Biol 2000, 132:365-386 [http://jura.wi.mit.edu/rozen/papers/rozen-and-skalet sky-2000-primer3.pdf].

28. Livak KJ, Schmittgen TD: Analysis of relative gene expression data using real-time quantitative PCR and the 2(delta delta C(T)) method. Methods 200I, 25:402-408.
Publish with Biomed Central and every scientist can read your work free of charge

"BioMed Central will be the most significant development for disseminating the results of biomedical research in our lifetime. "

Sir Paul Nurse, Cancer Research UK

Your research papers will be:

- available free of charge to the entire biomedical community

- peer reviewed and published immediately upon acceptance

- cited in PubMed and archived on PubMed Central

- yours - you keep the copyright
BioMedcentral 